\title{
Analysis on the Predicament of College Scientific Research Team Construction from the Perspective of Game Theory

\author{
Wei-min OUYANG ${ }^{1, a,{ }^{*}}$ and Hao-wen FENG $^{2, b}$ \\ ${ }^{1,2}$ Shanghai University of Political Science and Law

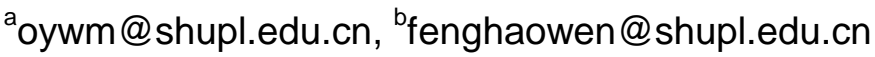

Keywords: Game Theory, Scientific Research Team, Strategy.

\begin{abstract}
The construction of scientific research team in colleges and universities is an important measure to improve the quality of scientific research in colleges and universities. In this paper, we treated the competition and cooperation in scientific research team as our research object, applying the method of game theory, according to the roles of different members, classified the game of internal competition and cooperation in scientific research team into three types: the game between strong member and ordinary member, the game between strong member and strong member, the game between ordinary member and ordinary member. From the perspective of game theory, we analyzed the strategies of the participants in the three cases, uncovered the predicament of college scientific research team construction, and proposed the corresponding strategies and suggestions through the analyses.
\end{abstract}

\section{Introduction}

In order to enhance the quality of scientific research in colleges and universities, the ministry of education carried out the "science and technology innovation team project", which requires colleges and universities to enhance the construction of scientific research team in colleges and universities [1,2, and 3]. Scientific research team is a special group of members, whose aim is to complete the scientific research construction and reform the pattern of scientist training.

The construction of scientific research team is an important measure to enhance the quality of scientific research in colleges and universities [1]. In the practice of scientific research team construction in domestic colleges and universities, since the teachers in colleges and universities are not full-time scientific research personnel, and also take the heavy daily teaching tasks, construction of scientific research team is temporarily built on the specific scientific research project, once the project is completed, the team will automatically be dissolved, the members of scientific research team in colleges and universities are not fixed, the structure of university scientific research team is relatively loose. Building of scientific research team in colleges and universities failed to make substantial progress, the overall level of scientific research team in colleges and universities is not very high.

Some experts and scholars attributed these phenomenon to self-interest, no overall situation consciousness, but have not make further analysis on the deep institutional reasons $[1,2,3,4$,$] . During the construction of scientific research team in colleges and$ universities, it is worth careful thorough research how to take effective incentive measures to promote team members within the members to engage in scientific research reform. 
In this paper, we treated the competition and cooperation in scientific research team in colleges and universities as the research object, applying the method of game theory [5], according to the role of members, classified the game of internal competition and cooperation in scientific research team into three types: the game between strong members and ordinary members, the game between strong members and strong members, the game between ordinary members and ordinary members. From the perspective of game theory, we analyzed the strategies of the participants in the three cases, uncovered the predicament of college scientific research team construction, and proposed the corresponding strategies and suggestions through the analyses.

\section{The Game between Strong Member and Ordinary Member}

The game has two participants, the one is a strong member, and the other is an ordinary member. All of them have two strategies of "positive" and "negative". Assume that the cost of scientific research team construction is 4, the income of scientific research team construction is 10, strong member will share 7 from 10, which expresses the reality of most strong members, that is, strong member has higher enthusiasm towards the construction of scientific research team, and can obtain better actual income after the success of scientific research team building, ordinary member will share 3 from 10 which expresses the reality of most ordinary members, that is, ordinary member has lower enthusiasm towards the construction of scientific research team, and can obtain poor actual income after the success of scientific research team building. Thus, there are four kinds of games between the strong member and ordinary member:

Case one: both of strong member and ordinary member select the strategy of "positive". In this case, the cost of scientific research team construction is 4 , the cost of construction shared by strong member A and B is 2, the income of strong member is 7 , and the income of ordinary member is 3 . So, the actual net income of strong member is 5 , the actual net income of ordinary member is 1 .

Case two: strong member selects the strategy of "positive", ordinary member selects the strategy of "negative". In this case, the strong member have to take all the cost of constructing scientific research team 4, while he will obtain the income of scientific research team construction 7 , and the actual net income of strong member is 3 . The ordinary member will obtain the income of scientific research team construction with no cost, and the actual net income of ordinary member is 3 .

Case three: strong member selects the strategy of "negative", ordinary member selects the strategy of "positive". In this case, the ordinary member has to take all the cost of constructing scientific research team 4 , while he will obtain the income of scientific research team construction 3 , and the actual net income of ordinary member is -1 . The strong member will obtain the income of scientific research team construction with no cost, the actual net income of strong member is 7 .

Case four: both of strong member and ordinary member select the strategy of "negative". In this case, both of strong member and ordinary member have no cost of constructing scientific research team, and have no the income of scientific research team construction, both of their the actual net incomes are 0 .

Based on the above descriptions, the game's payoff matrix is described in table 1. 
Table 1 . The game's payoff matrix between strong member and ordinary member

\begin{tabular}{|l|l|l|l|}
\hline & \multicolumn{3}{|c|}{ ordinary member } \\
\hline \multirow{3}{*}{ strong member } & & positive & negative \\
\cline { 2 - 4 } & positive & 5,1 & $\underline{3}, \underline{3}$ \\
\cline { 2 - 4 } & negative & $\underline{7},-1$ & $0, \underline{0}$ \\
\hline
\end{tabular}

According to the above game's payoff matrix, we discuss the strategy choice of strong member and ordinary member respectively.

First, consider the strategy choices of strong member. If ordinary member selects "positive" strategy, then the profit of strong member in choosing "positive" strategy is 5 and the profit of strong member in choosing "negative" strategy is 7 , because $7>5$, the choice of "negative" strategy is strong member' s optimal reaction under ordinary member taking "positive" strategy; If ordinary member selects "negative" strategy, then the profit of strong member in choosing "positive" strategy is 3, and the profit of strong member in choosing "negative" strategy is 0 , because $3>0$, the choice of "negative" strategy is strong member' s optimal reaction under ordinary member taking "negative" strategy. Therefore, strong member has no dominant strategy in the game between strong member and ordinary member, whose optimal reaction depends on the strategies taken by ordinary member. If ordinary member take "positive" strategy, strong member had better to take "negative" strategy; if ordinary member take "negative" strategy, strong member had better to take "positive" strategy.

Second, consider the strategy choices of ordinary member. If strong member "positive" strategy, then the profit of ordinary member in choosing "positive" strategy is 1 , and the profit of ordinary member in choosing "negative" strategy is 3, because $3>1$, the choice of "negative" strategy is ordinary member' $s$ optimal reaction under strong member taking "positive" strategy; if strong member selects "negative" strategy, then the profit of ordinary member in choosing "positive" strategy is -1 , and the profit of ordinary member in choosing "negative" strategy is 0 , because $0>-1$, the choice of "negative" strategy is ordinary member' s optimal reaction under strong member taking "negative" strategy. Therefore, whether the strong member's strategy is "positive" or "negative", the dominant strategy of ordinary member in the game between strong member and ordinary member is "negative".

Because the dominant strategy of ordinary member in the game between strong member and ordinary member is "negative", ordinary member will always select "negative" strategy in this game, and strong member will have to select "positive" strategy. So, the Nash equilibrium of the game is ("positive", "negative").

Therefore, the game structure is the classic "boxed pigs game" model, whose Nash equilibrium of the game is ("positive", "negative"), that is to say, ordinary member must select "negative" strategy, and strong member has to select "active" strategy. The strong member has to act as the role of "big pig", to undertake the responsibility of scientific research team construction. And meanwhile the ordinary member will freely share the results of the construction of the strong member, just act as the small pig in "boxed pigs game" take a ride of big pig.

In the boxed pig' s game, the big pig selects "tread", on the subjective, is for their own interests, but on the objective, the small pig can be free to enjoy the benefits. In the boxed pigs game between strong member and ordinary member, both parties as a rational person, strong member in their own needs to undertake the task of building the scientific research team, ordinary member of scientific research team construction is not interested, lacks 
motivation, he need not to make an effort, but can freely share the achievements of the construction of the strong member.

\section{The Game between Strong Member and Strong Member}

Like the last section, we assume that the cost of scientific research team construction is 4 , the income of scientific research team construction is 10, and each of the two strong members will share 5 from 10, respectively. Thus, there are four kinds of games between the strong member $\mathrm{A}$ and strong member B:

Case one: both of strong members A and B select "positive" strategy. In the case, the cost of scientific research team construction is 4 , the cost of constructing scientific research team shared by strong member $\mathrm{A}$ and $\mathrm{B}$ is 2 , and the income of strong member from scientific research team construction is 5 . Therefore, the actual net income of the both participants is 3 .

Case two: strong member A selects the strategy of "positive", strong member B selects the strategy of "negative". In this case, the strong member A has to take all the cost of constructing scientific research team 4 , while he will obtain the income of scientific research team construction 5, and the actual net income of strong member $A$ is 1 . The strong member B will obtain the income of scientific research team construction with no cost, which is 5 , and the actual net income of strong member B is 5 .

Case three: strong member A selects the strategy of "negative", strong member B selects the strategy of "positive". In this case, the strong member B has to take all the cost of constructing scientific research team 4, while he will obtain the income of scientific research team construction 5 , and the actual net income of strong member B is 1 . The strong member A will obtain the income of scientific research team construction with no cost, which is 5 , and the actual net income of strong member is 5 .

Case four: both of strong member A and B select the strategy of "negative". In this case, both of the strong members make no efforts on scientific research team construction, and have no the income of scientific research team construction, both of their actual net incomes are 0 .

Based on the above description, the game's payoff matrix is described in table 2.

Table 2: The game's payoff matrix between strong members

\begin{tabular}{|l|l|l|l|}
\hline & \multicolumn{3}{|c|}{ strong member B } \\
\hline \multirow{3}{*}{ strong member A } & & positive & negative \\
\cline { 2 - 4 } & positive & 3,3 & 1,5 \\
\cline { 2 - 4 } & negative & 5,1 & 0,0 \\
\hline
\end{tabular}

Consider the strategy choices of strong member A. If strong member B selects "positive" strategy, then the profit of strong member A in choosing "positive" strategy is 3 and the profit of strong member A in choosing "negative" strategy is 5, because 5>3, the choice of "negative" strategy is optimal reaction of strong member A under strong member B taking "positive" strategy; If strong member B selects "negative" strategy, then the profit of strong member A in choosing "positive" strategy is 1 , and the profit of strong member A in choosing "negative" strategy is 0 , because $1>0$, the choice of "positive" strategy is optimal reaction of strong member A under strong member B taking "negative" strategy. Therefore, strong member A has no dominant strategy in the game between strong member A and 
strong member $\mathrm{B}$, whose optimal reaction depends on the strategies taken by strong member B. If strong member B takes "positive" strategy, strong member A had better to take "negative" strategy; if strong member B take "negative" strategy, strong member A had better to take "positive" strategy.

By considering the strategy choices of strong member B as above, strong member B has no dominant strategy in the game between strong member $\mathrm{A}$ and strong member $\mathrm{B}$, whose optimal reaction depends on the strategies taken by strong member A. If strong member A take "positive" strategy, strong member B had better to take "negative" strategy; if strong member A take "negative" strategy, strong member B had better to take "positive" strategy.

Therefore, the game structure is the classic "chicken game" model, which has no pure Nash equilibrium, but has two mixed strategy Nash equilibrium ("positive", "negative") and ("negative", "positive"), that is to say, if one party selects "positive", the other party will select "negative"; if one party selects "negative", the other party will select "positive".

In a chicken game, both parties have no common interests, if one party insisted on game, the other is difficult to withdraw from the game. In the chicken game between strong members, both parties as a rational person, any one party must hope the other party to select "positive" strategy to build scientific research team, and he selects "negative" strategy, so as to share the achievements of scientific research team construction.

By taking the risk of choosing "negative" strategy, the winner is to establish his own "happiness" on the "pain" of other side.

However, in the real environment, because the strong members have a high requirement of scientific research team construction, and their self expectation are very high, although at the beginning of the game will take "negative" strategy, but after a period of time, they will be willing to select "positive" strategy to build scientific research team. The theoretical mixed strategy Nash equilibrium is often not true in reality choice.

\section{The Game between Ordinary Member and Ordinary Member}

We also assume that the cost of scientific research team construction is 4 , the income of scientific research team construction is 6 , and each of the two ordinary members will share 3 from 6 , respectively. Thus, there are four kinds of games between the ordinary member A and ordinary member B:

Case one: both of ordinary members A and B select the strategy of "positive". In this case, the cost of scientific research team construction is 4, the cost of constructing scientific research team shared by ordinary member $A$ and $B$ is 2 , and the income of ordinary member is 3. So, the actual net income of the both participants is 1 .

Case two: ordinary member A selects the strategy of "positive", ordinary member B selects the strategy of "negative". In this case, the ordinary member A has to take all the cost of constructing scientific research team 4, while he will obtain the income of scientific research team construction 3 , and the actual net income of ordinary member $A$ is 1 . The ordinary member B will obtain the income of scientific research team construction with no cost, which is 3 , and the actual net income of ordinary member B is 3 .

Case three: ordinary member A selects the strategy of "negative", ordinary member B selects the strategy of "positive". In this case, the ordinary member B has to take all the cost of constructing scientific research team 4 , while he will obtain the income of scientific research team construction 3 , and the actual net income of ordinary member B is -1 . The 
ordinary member A will obtain the income of scientific research team construction with no cost, which is 3 , and the actual net income of ordinary member is 3 .

Case four: both of ordinary member A and ordinary member B select the strategy of "negative". In this case, both of the ordinary members have no cost of scientific research team construction, and have no the income of scientific research team construction, both of their actual net incomes are 0 .

Based on the above description, the game's payoff matrix is described in table 3.

Table 3: The game's payoff matrix between ordinary members

\begin{tabular}{|l|l|l|l|}
\hline & \multicolumn{3}{|c|}{ ordinary member B } \\
\hline \multirow{3}{*}{ ordinary member A } & & positive & negative \\
\cline { 2 - 4 } & positive & 1,1 & $-1,3$ \\
\cline { 2 - 4 } & negative & $3,-1$ & 0,0 \\
\hline
\end{tabular}

Consider the strategy choices of ordinary member A. If ordinary member B selects "positive" strategy, then the profit of ordinary member A in choosing "positive" strategy is 1 and the profit of ordinary member $A$ in choosing "negative" strategy is 3 , because $3>1$, the choice of "negative" strategy is optimal reaction of ordinary member A under ordinary member B taking "positive" strategy; If ordinary member B selects "negative" strategy, then the profit of ordinary member A in choosing "positive" strategy is -1 , and the profit of ordinary member A in choosing "negative" strategy is 0 , because $0>-1$, the choice of "negative" strategy is optimal reaction of ordinary member A under ordinary member B taking "negative" strategy. Therefore, whether the strategy of ordinary member B is "positive" or "negative", the dominant strategy of ordinary member A in the game between ordinary member A and ordinary member B is "negative".

By considering the strategy choices of ordinary member B in the same way, we can know, "negative" strategy is also the dominant strategy of ordinary member B in the game between ordinary member A and ordinary member B.

Therefore, the game structure is the classic " prisoner's dilemma" model, which has a pure Nash equilibrium ("negative", "negative"), that is to say, both of ordinary member A and B will select "negative", although the total profit of ordinary member A and B in choosing "positive" strategy is not only lower than the total income of both in choosing "positive" strategy, but also lower than the total profit of only one ordinary member to select "positive" strategy, and thus fall into the famous "the prisoner's dilemma". Based on individual rationality, both of ordinary member A and B made their choices in order to maximize the interests of the individual, but in fact, they got the worst result, which not only injure their own interests, but also injure the collective interests.

\section{Conclusions and Suggestions}

Based on the above analysis, the competition and cooperation within the scientific research team can come down to three different types of game model respectively. Whether boxed pigs game, or a coward or prisoner's dilemma game, the choices of members are the result of the rational choice in the real environment. To conquer the predicament of the scientific research team construction, college and university must manage to adjust the game payment function about the both sides of the game. For example, college and university can increase the profit on scientific research team construction in order to enhance the positive 
motivation for building scientific research team. Since college and university can adjust the profits of participants in the scientific research team construction, the Nash equilibrium of the game can reach the convergence to the ideal win-win cooperation state. Therefore, all kinds of members have intrinsic motivation to actively take part in the construction of scientific research team.

Colleges and universities should be to design and adjust various mechanisms in scientific research team construction, such as incentive mechanism, sharing mechanism, responsibility sharing mechanism, effectiveness evaluation mechanism.

Only to reform and innovate the existing mechanism for building scientific research team, may inspire the enthusiasm for members to participate in building scientific research team, and may realize the goal of improving the quality of scientific research in colleges and universities.

\section{References}

[1] Qian Yang, A Study on the Organization and Management of University's Scientific Research Team, In the Proceedings of The 6th International Conference on Education, Management, Computer and Society (EMCS 2016), pp.1481-1483, January 2016.

[2] Wang Yiran, Chen Shijun, Zhang haiyan, et al., Study on Theoretical problems in the Construction of Creativity Team in University, Journal of Science \& Technology Progress and policy, Vol.23, pp.194-197, Aug. 2007.

[3] Fang Yong, Wang Mingming, Liu Mu, Under Innovation Angle to Discuss Organizational Structure Design of University' s Science and Research Team, Journal of Science \& Technology Progress and policy, Vol.25, pp.190-183, May 2008.

[4] E.R.Wright, L.A.Russell, te al., Impact of Team Structure on Achieving Treatment Goals in System of Care, Journal of Emotional and behavioral Disorder, Vol.14, pp. 240-250, Winter 2006.

[5] Wu guangmou, Lv zhouxiang, Principles and Application of Game Theory, Southeast University Press, Mar. 2009. 